\title{
A review of enhanced paramedic roles during and after hospital handover of stroke, myocardial infarction and trauma patients
}

Darren Flynn ${ }^{1 *} \mathbb{D}$, Richard Francis ${ }^{2}$, Shannon Robalino ${ }^{3}$, Joanne Lally ${ }^{1}$, Helen Snooks ${ }^{4}$, Helen Rodgers ${ }^{2}$, Graham McClelland ${ }^{5}$, Gary A. Ford ${ }^{6}$ and Christopher Price ${ }^{2}$

\begin{abstract}
Background: Ambulance paramedics play a critical role expediting patient access to emergency treatments. Standardised handover communication frameworks have led to improvements in accuracy and speed of information transfer but their impact upon time-critical scenarios is unclear. Patient outcomes might be improved by paramedics staying for a limited time after handover to assist with shared patient care. We aimed to categorize and synthesise data from studies describing development/extension of the ambulance-based paramedic role during and after handover for time-critical conditions (trauma, stroke and myocardial infarction).
\end{abstract}

Methods: We conducted an electronic search of published literature (Jan 1990 to Sep 2016) by applying a structured strategy to eight bibliographic databases. Two reviewers independently assessed eligible studies of paramedics, emergency medical (or ambulance) technicians that reported on the development, evaluation or implementation of (i) generic or specific structured handovers applied to trauma, stroke or myocardial infarction (MI) patients; or (ii) paramedic-initiated care processes at handover or post-handover clinical activity directly related to patient care in secondary care for trauma, stroke and MI. Eligible studies had to report changes in health outcomes.

Results: We did not identify any studies that evaluated the health impact of an emergency ambulance paramedic intervention following arrival at hospital. A narrative review was undertaken of 36 studies shortlisted at the full text stage which reported data relevant to time-critical clinical scenarios on structured handover tools/protocols; protocols/enhanced paramedic skills to improve handover; or protocols/enhanced paramedic skills leading to a change in in-hospital transfer location. These studies reported that (i) enhanced paramedic skills (diagnosis, clinical decision making and administration of treatment) might supplement handover information; (ii) structured handover tools and feedback on handover performance can impact positively on paramedic behaviour during clinical communication; and (iii) additional roles of paramedics after arrival at hospital was limited to 'direct transportation' of patients to imaging/specialist care facilities.

Conclusions: There is insufficient published evidence to make a recommendation regarding condition-specific handovers or extending the ambulance paramedic role across the secondary/tertiary care threshold to improve health outcomes. However, previous studies have reported non-clinical outcomes which suggest that structured handovers and enhanced paramedic actions after hospital arrival might be beneficial for time-critical conditions and further investigation is required.

Keywords: Systematic review, Paramedics, Extended role, Stroke, Acute myocardial infarction, Trauma

\footnotetext{
*Correspondence: darren.flynn@ncl.ac.uk

'Institute of Health and Society, Newcastle University, Baddiley-Clark Building,

Richardson Road, Newcastle upon Tyne NE2 4AX, United Kingdom

Full list of author information is available at the end of the article
} 


\section{Background}

Evidence-based care standards emphasise the importance of early recognition, appropriate conveyance and coordinated care for patients presenting as medical emergencies [1]. This is particularly important for optimising outcomes amongst those conditions with time-critical treatments, notably trauma, myocardial infarction (MI) and acute stroke [2]. Ambulance-based paramedics have a critical role providing rapid pre-hospital assessment, triage and expediting access to these emergency treatments. However, a review of 21 clinical handover studies raised concerns about the quality of communication and information exchange between pre-hospital and hospital staff, particularly when under time pressure due to service demands [2-4]. Structured handover frameworks have been shown to improve the accuracy and speed of information transfer between paramedic and emergency department (ED) staff, [5] but it is unclear whether condition-specific versions could further improve care and health outcomes for time-critical conditions.

In clinically complex situations, patient outcomes might be improved further if paramedics and/or emergency medical technicians continued to contribute actively towards care alongside the emergency hospital team for a limited time after handover, such as assisting with airway management or intravenous access. Following handover of standard clinical information, paramedics do not routinely continue to be part of patient's ongoing care in the ED, coronary care, stroke unit or other appropriate specialist treatment facility in secondary care. However, they might possess relevant skills at a time when the hospital team can be subject to competing demands, which could contribute further towards faster access to emergency treatments. Achieving optimal health outcomes may require development of enhanced clinical roles which transgress traditional boundaries.

In order to summarise the current evidence describing the impact of enhanced paramedic processing of emergency conditions with time-dependent treatment outcomes (i.e. trauma, stroke and MI), we undertook a review of literature reporting the clinical effectiveness of (i) structured paramedic/emergency medical technician handovers to emergency hospital teams, and (ii) paramedics/emergency medical technicians or ambulance technicians continuing to contribute actively towards care alongside the emergency hospital team after handover (i.e. an enhanced/expanded role across the threshold of secondary care).

In order to provide additional context in terms of process/operational data relevant to time-critical clinical conditions that are the current focus of service restructuring to facilitate care pathways to improve health outcomes, a secondary objective was to categorize and narratively describe relevant studies which did not meet the full inclusion criteria but explored either: (i) development and evaluation of novel structured handover tools or protocols; (ii) protocols or enhanced skills to improve handover; or (iii) protocols or enhanced skills leading to a change in in-hospital transfer location.

\section{Methods}

The review process adhered to a published protocol [6] and reporting guidelines of the Preferred Reporting Items for Systematic Reviews and Meta-analyses (PRISMA) statement [7].

\section{Study designs}

Experimental, quasi-experimental and observational research studies, including qualitative and mixed methods studies were eligible for inclusion. Eligible studies involved paramedics, emergency medical technicians (EMTs) or ambulance technicians (including the armed forces) that reported on the development, evaluation or implementation of (i) novel structured handovers to hospital-based physicians for acute stroke, acute MI or trauma patients; or (ii) new processes at handover or post-handover clinical activity that actively contributed towards a patient's care alongside the emergency hospital team for a limited time after handover in the secondary care setting for acute stroke, acute MI or trauma. Trauma patients were defined as those with any lifethreatening injury requiring urgent treatment within $24 \mathrm{~h}$. Eligible studies were also required to assess changes in health outcomes (e.g. survival, quality of life or functional status at $24 \mathrm{~h}$ or discharge).

Studies were excluded if the focus was on pre-hospital activity only (e.g. telephone communication, implementation of Advanced Cardiac Life Support algorithms), protocol driven ambulance redirection (e.g. to a different hospital or specialist hub), non-clinical activity (e.g. evaluation of electronic record systems) or involved transportation with an accompanying physician (i.e. patient care was medically-driven from the outset). Paramedics/emergency medical technicians with extended community roles designed to reduce hospital admissions and those already based in hospital (i.e. working routinely in an emergency department) were also excluded if they did not involve an interaction with a hospital for avoiding or facilitating emergency admissions.

\section{Search strategy}

A structured search strategy (MeSH terms and keywords) designed by an experienced information scientist (SR) was applied to bibliographic databases (MEDLINE, PubMed, HMIC - Health Management Information Consortium, Cochrane HTA, Cochrane Central, EMBASE, ASSIA and PsycInfo. Main search terms included ambulance, EMIS, paramedic, pre-hospital, myocardial infarction, stroke and 
trauma (see Appendix 1 for an example of the full search strategy in MEDLINE). The design, treatments and professional roles within routine emergency medical services have evolved significantly since the 1980s; therefore the search was restricted to studies published from Jan 1990 to Sep 2016. No restrictions were placed on country of origin, but searches were restricted to abstracts published in English. Hand-searching of reference lists and citation searching of eligible studies was also undertaken (including relevant reviews identified by the search strategy). Grey literature was identified from contact with content experts (NASMED, College of Paramedics).

\section{Study selection, data extraction and assessment of methodological quality}

Two reviewers (DF and RF) independently assessed the titles and abstracts retrieved via the search strategy (stage 1) and independently assessed the retained full text studies using a study selection form (Appendix 2). Disagreements at the full text stage were resolved via discussion or by consulting with a third member of the review team $(\mathrm{CP})$.

Two authors independently captured information on study characteristics (study design, participants, context, any new care processes undertaken by paramedics/EMTs at handover/post-handover and outcomes) and assessed methodological quality according to the frameworks developed by the Cochrane Collaboration $[8,9]$.

\section{Data synthesis}

A high degree of heterogeneity between study designs and outcomes was expected, therefore no sensitivity analyses were planned, and a narrative approach was used to synthesise the findings of included studies.

\section{Results}

A total of 17,972 hits were generated by the search strategy (Fig. 1). Of the 43 full text articles identified following initial screening, seven were excluded for not involving paramedics/emergency medical technicians or ambulance technicians [10], focusing on accuracy of patient information transfer from pre-hospital to the ED [11], duplicating information [12, 13] in published papers $[5,14]$ or did not involve a new interaction between the paramedic and hospital or change in patient admission destination [14-16]. Although relevant as interventions that could be evaluated for improving health outcomes, none of the remaining 36 articles fulfilled all of the review criteria, in particular omission of an evaluation of health outcomes in the context of stroke, MI or trauma. These 36 studies have been summarised into three categories for narrative synthesis (Table 1): structured handover tools/protocols, protocols and enhanced skills to improve handover, and protocols or enhanced skills leading to a change in in-hospital transfer location.

Seven studies described approaches to improve clinical handover between paramedics/EMTs and hospital-based ED clinicians, but did not focus specifically on stroke, MI, or trauma patients or include a health outcome [5, 17-22]. For trauma patients, an uncontrolled before and after design [20] was used to evaluate the impact of a web-based, educational intervention designed to enhance paramedic verbal communication skills during handover to hospital physicians. Research associates collected recordings and made notes of patient handover conversations and then interviewed physicians to assess their recall of details provided by paramedics. Overall physicians recalled 36\% of paramedic verbal reports, but pre- and post-intervention levels of recall by physicians failed to reach statistical significance (33 and $38 \%, p=0.16$ ). In a similar study, a simple training intervention ( $3 \mathrm{~h}$ in duration) consisting of five rules of communication, simulated case scenarios and a handover protocol (inventory, medical history, clinical findings and actions) reported that the frequency of negative communication events decreased from 3.9 per scenario before training to 1.8 after training [22]. A focus group study [19] identified the following professional, structural and interpersonal factors related to improvement of trauma handovers from the perspective of paramedics $(N=48)$ : direct communication with ED clinician who was responsible for care of the patient; inter-disciplinary understanding and feedback to nurture a shared understanding of paramedic and ED roles; standardising selected aspects of the handover process; and use of technology to facilitate information exchange. However there was no evaluation of these principles in practice.

Four studies reported on the development of new generic protocols/checklists to improve handover quality $[5,17,18$, 23]. In a grounded theory study [18] ten paramedics and 17 hospital-based trauma clinicians were interviewed regarding adaptation of the MIST tool (Mechanism, Injury Pattern, Signs and Treatment) and highlighted the importance of concise delivery of structured information to inform immediate treatment, and hospital pre-notification. A prospective pre-test post-test study [23] evaluated the effectiveness of an e-learning tool for improving adherence to DeMIST (Demographics, Mechanism of Injury/illness, Injury or Illness found or suspected, Signs, Treatment given), but failed to show any changes in use or adherence to the sequence of this tool for structuring pre-hospital notification and handover. An Australian study used videoreflexive ethnography [5] to demonstrate the feasibility of improving handover communication between paramedics and ED staff using a standard order of information (IMST AMBO: Identification, Mechanism, Injuries, Signs, Treatment, Allergies, Medications, Background, Other) as indicated by an increase in the amount of consistently ordered 


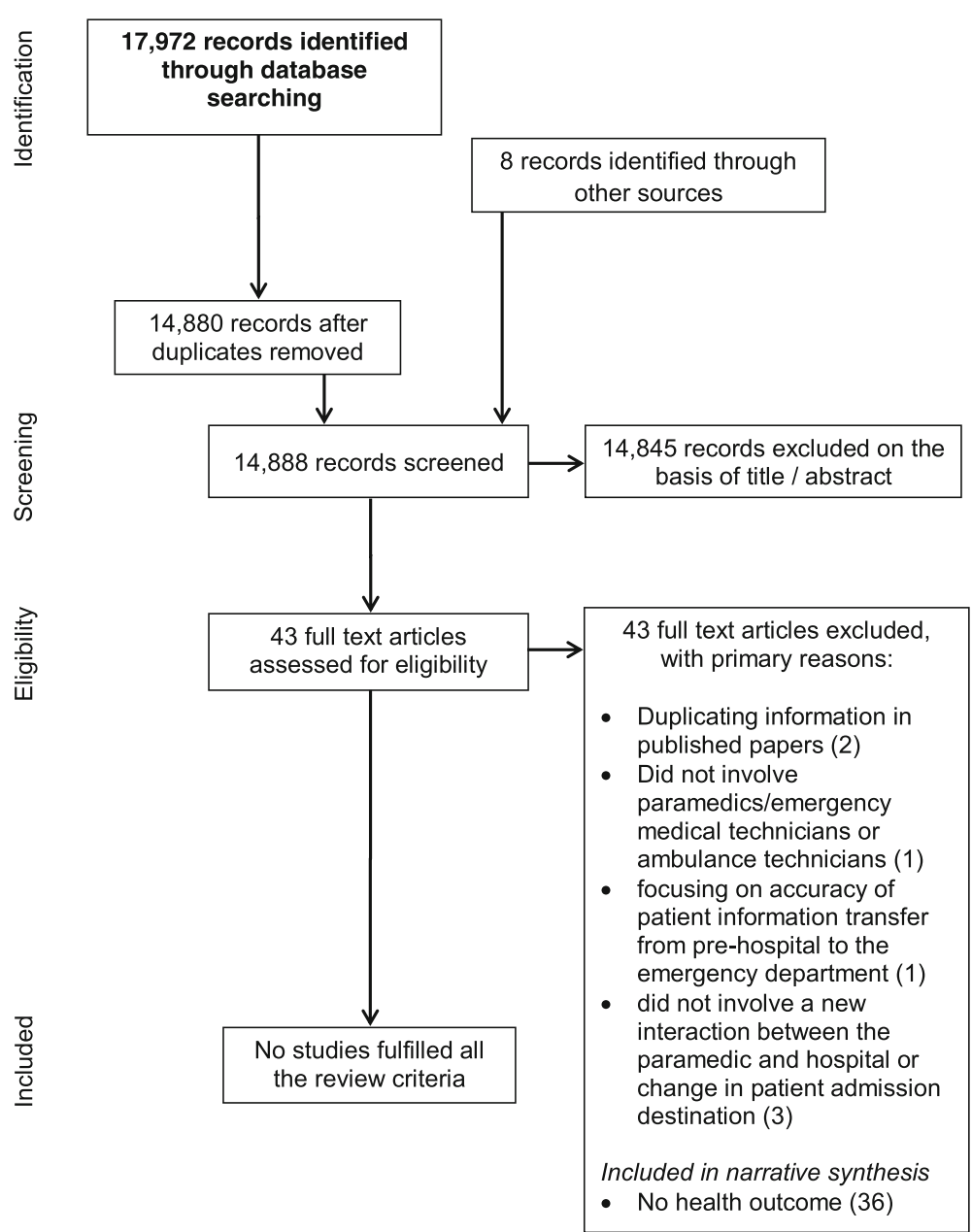

Fig. 1 PRISMA flow diagram of the process used to identify studies

information per handover, fewer clarifications from ED staff, shorter handover duration, and increased eye contact. Another observational study using video assessment was unable to show better communication from applying a structured format (ISBAR: Identification, Situation, Background, Assessment and Request) during simulated handovers [17]. This may reflect the basic content of the information conveyed and the restrictive nature of the short simulations.

One study focused specifically on the role of paramedics in relation to patient medication during handover [21], which reported a statistically significant increase in the percentage of patients' who were reconciled with their own medication in the ED (67\% at pre-intervention versus $87 \%$ post-intervention) and a reduced percentage of errors in regular medications prescribed (18.9\% pre-intervention versus $8.8 \%$ post-intervention).

Sixteen studies reported on paramedic actions for trauma, stroke and MI patients that were in addition to standard handover [24-39]. They did not directly provide patient care, and the paramedic role was restricted to prehospital activation $[25,37]$ or transportation of patients to condition-specific specialist facilities such as catheterisation laboratory for MI patients [26, 28, 30-33, 38], radiology for acute stroke patients [24, 27, 34, 36, 39], radiology for trauma (hip fracture) patients [35], or a pacing centre for patients with complete heart block [29]. In one study paramedics were also responsible for delivery of blood test results of trauma (hip fracture) patients to the hospital laboratory [35]. The primary aim of these studies was to improve process measures, with one study reporting improved call to door times [34], two improved door to imaging times $[24,27]$ and eight improved door to treatment times [26, 27, 30-34, 36, 38].

Several studies reported positive findings in hospital resulting from 'advanced paramedic' skills but these had only been applied during the pre-hospital phase such as clinical diagnoses of ST elevation MI [30-32] and paramedic-administered morphine or atropine [40] and fibrinolytic treatment $[41,42]$. 


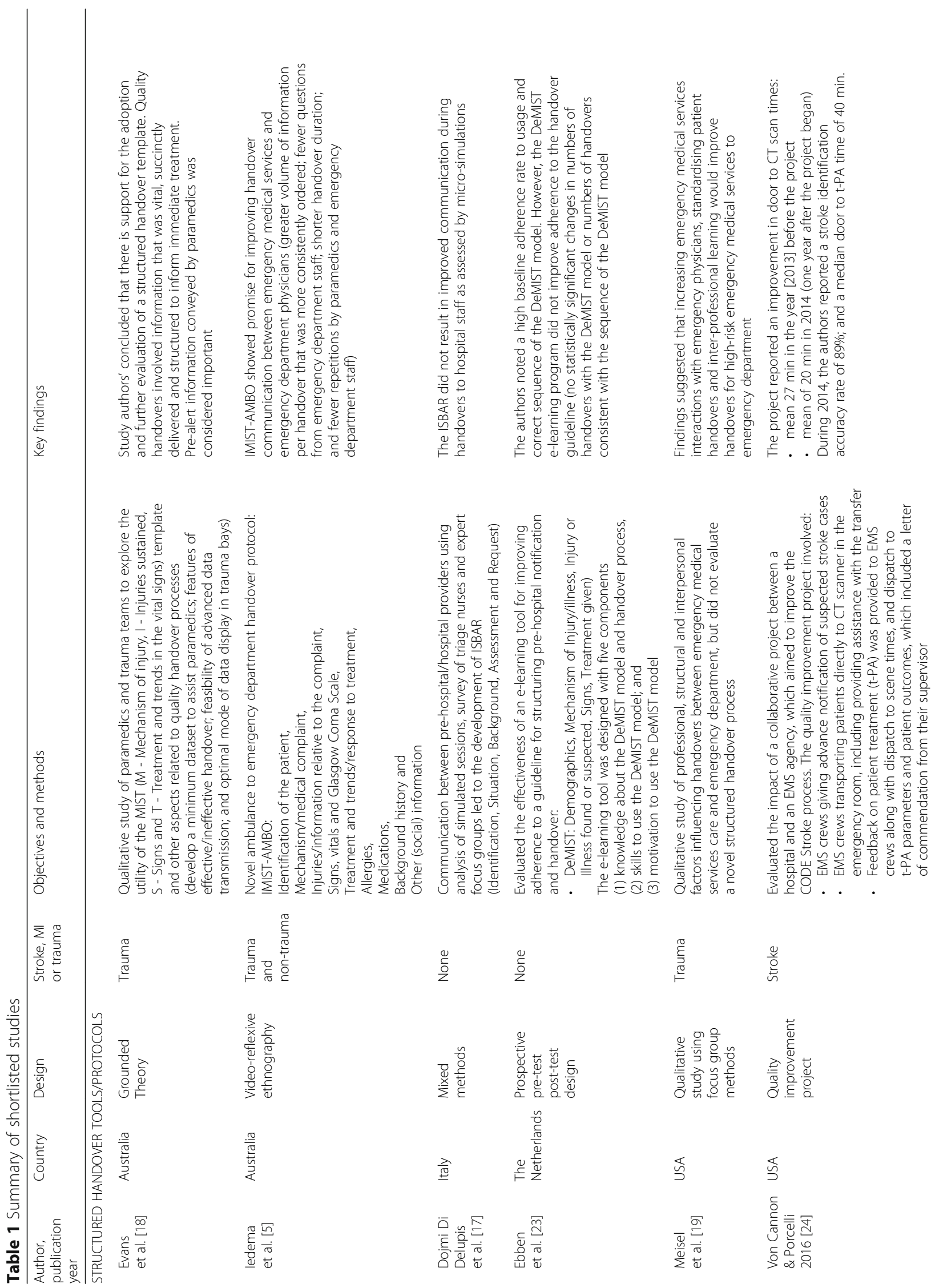




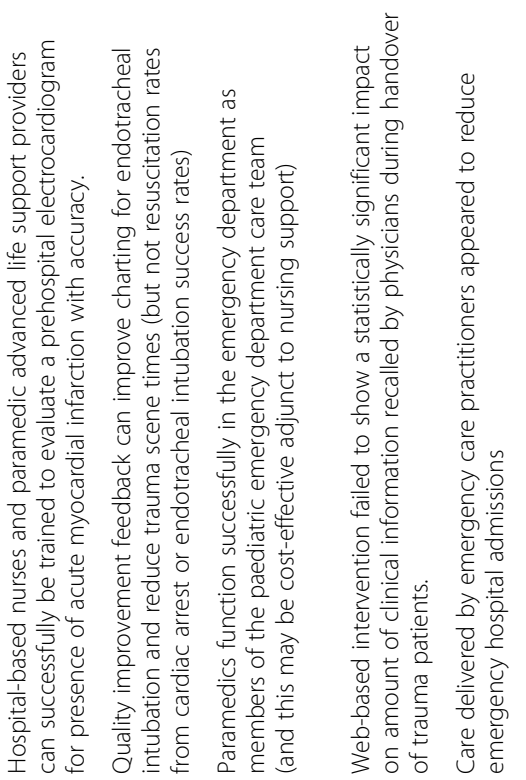

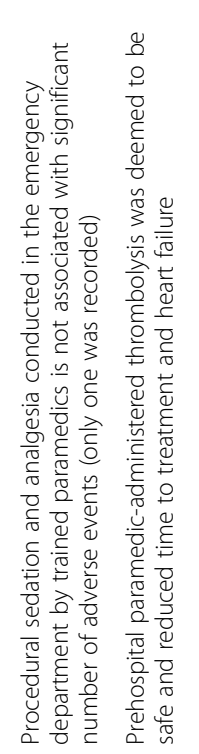
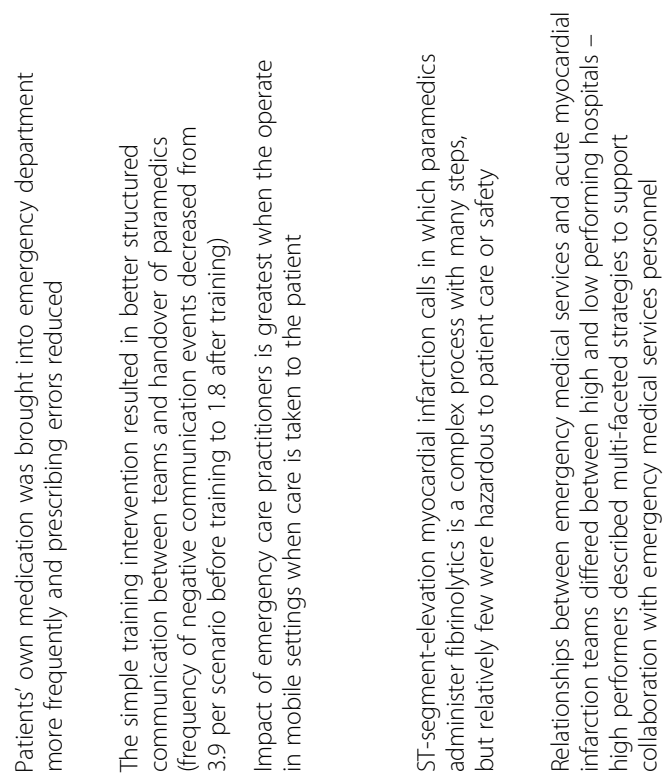

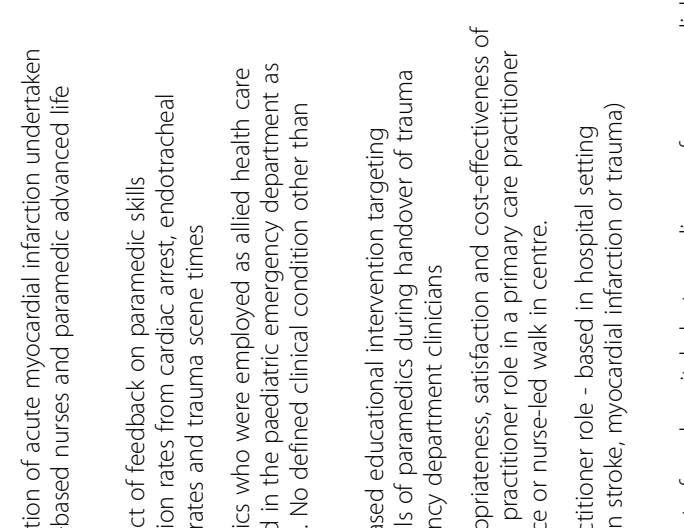

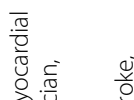

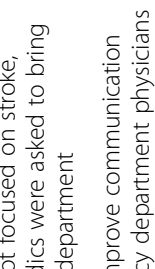

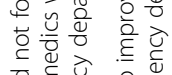

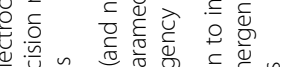

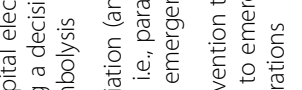

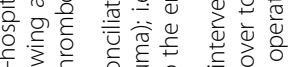

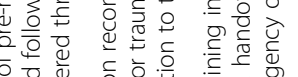

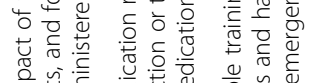

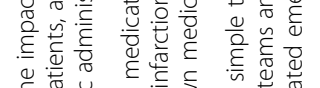

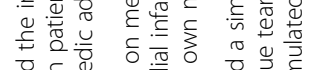

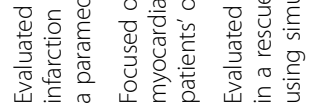

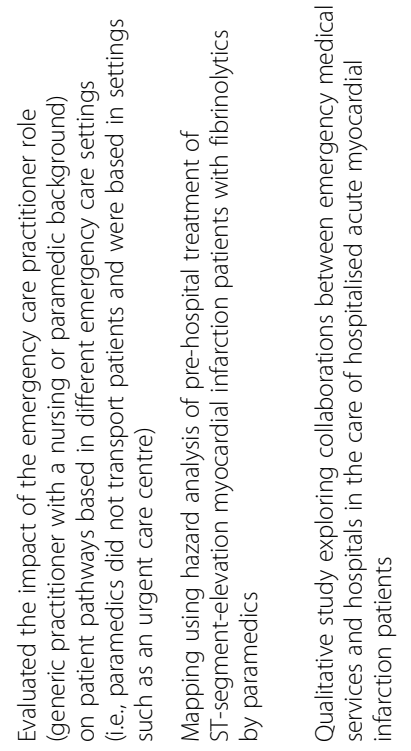

จे

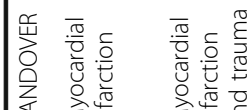

垔

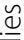

㝕

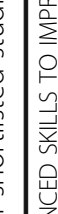

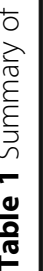

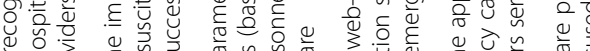

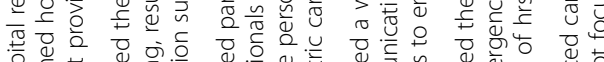

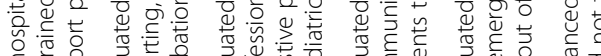

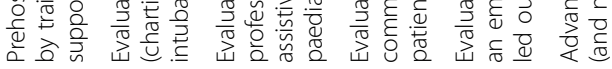

1

高

产

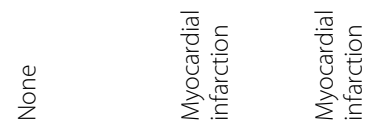

穵

ह

影

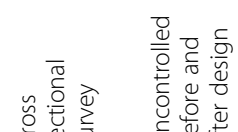

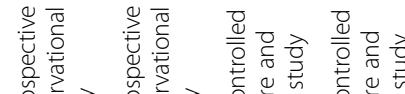

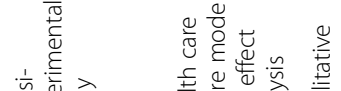

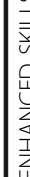

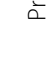
赵

要

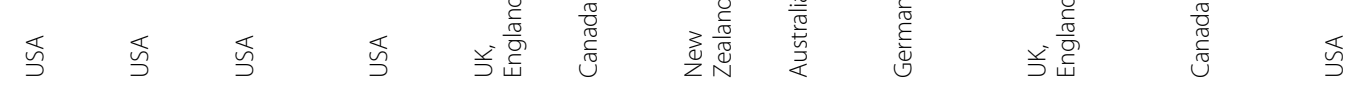

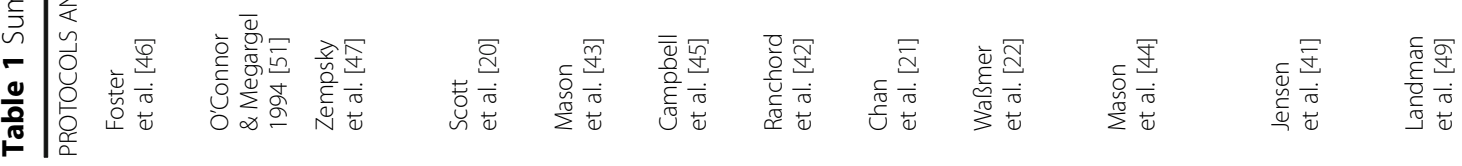




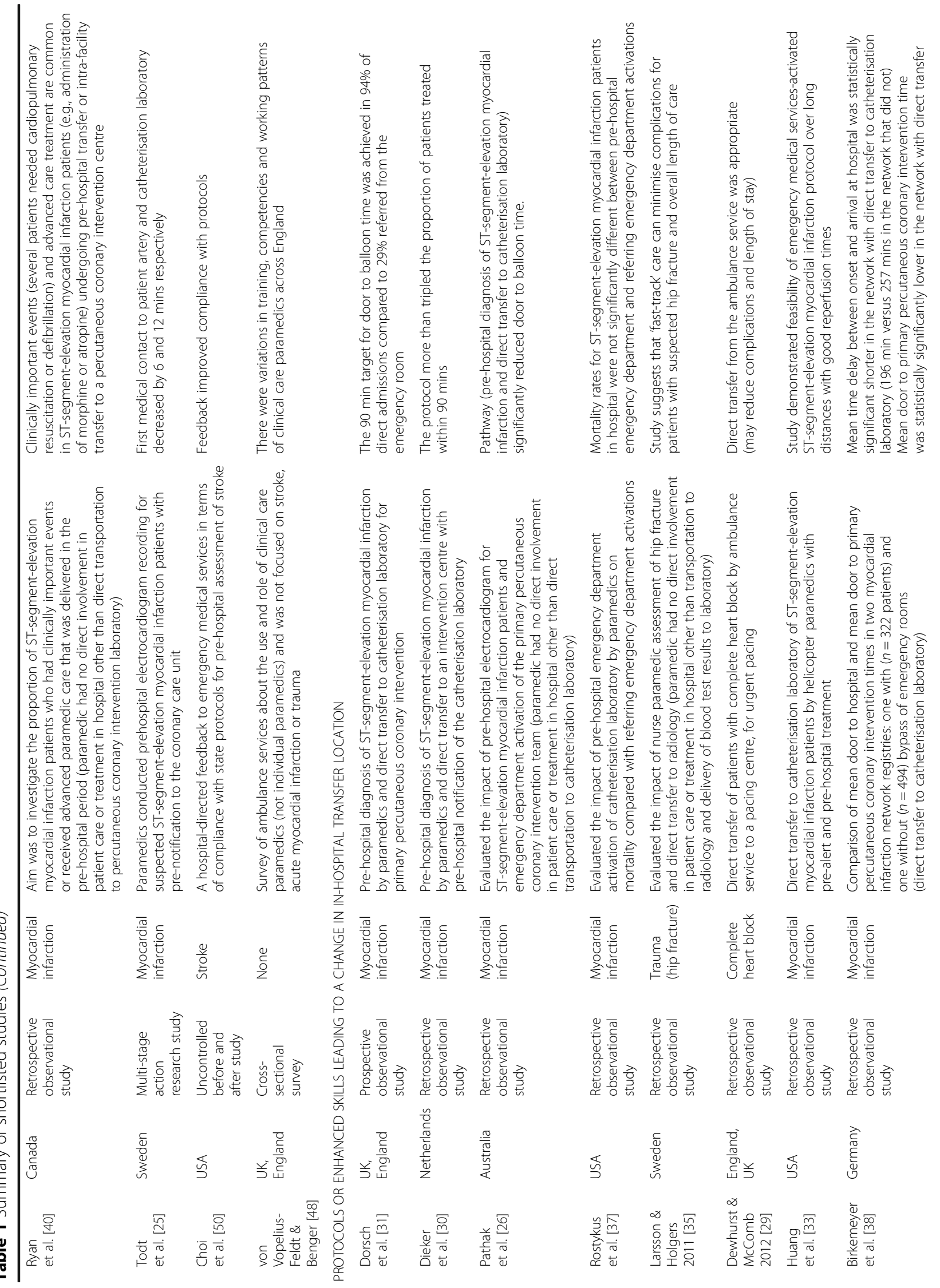




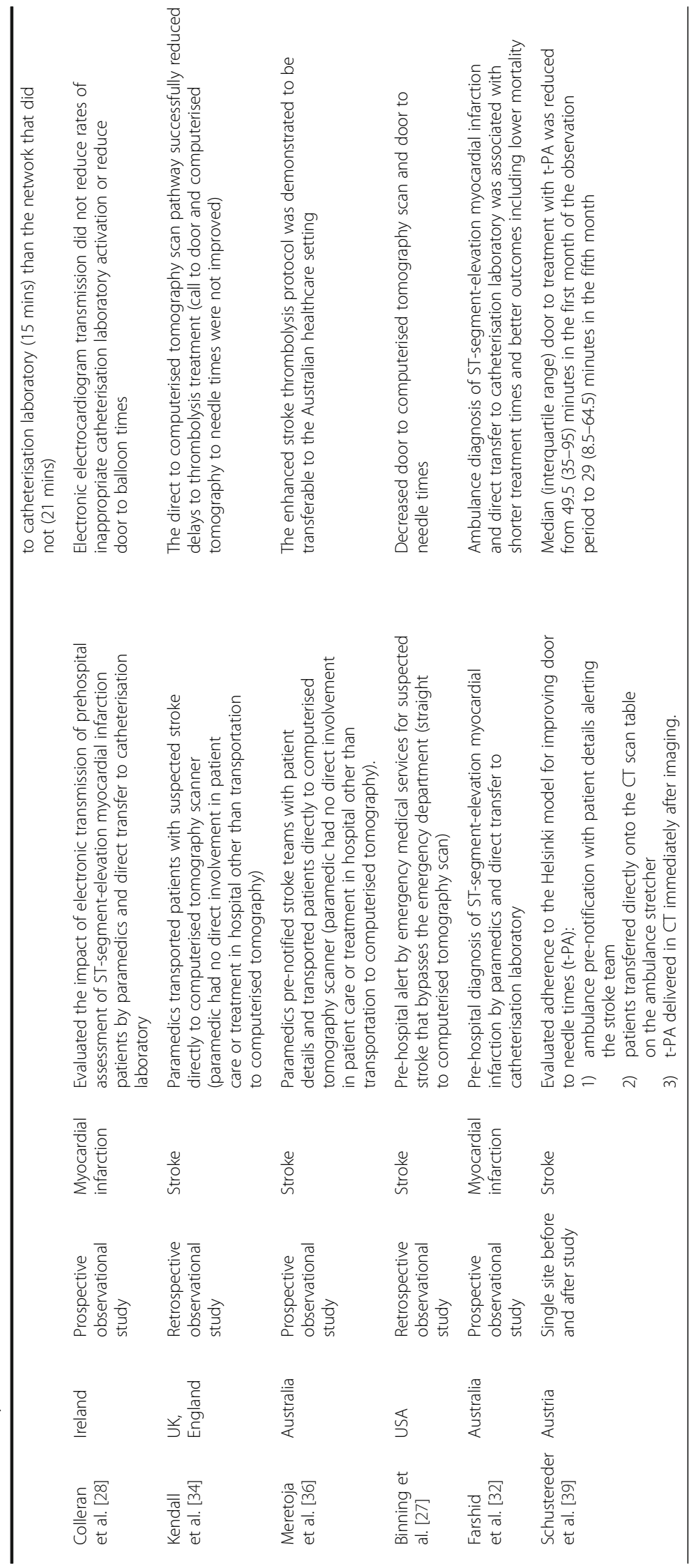


Amongst the studies were descriptions of successfully implemented novel assistive paramedic roles within primary care settings/community-based walk-in centres [43, 44], but with the intention of reducing emergency admissions to the ED. Three studies reported on paramedics based in hospitals providing assistance to clinical teams by administration of procedural sedation and analgesia [45], evaluating a prehospital electrocardiogram (ECG) for presence of MI [46] and assessment of paediatric patients [47]. The range of such activities undertaken by paramedics in the UK National Health Service (NHS) was described in a survey [48], which highlighted the variation in training, competencies and working patterns, but did not identify any handover or post-handover paramedic-led interventions for trauma, stroke and MI.

Feedback to paramedics by the hospital team was considered important but was not compared against future health outcomes. 148 hospital staff involved with MI patients were interviewed about their relationship with EMS services [49] and those from high performing hospitals (upper 5\% of hospitals based on 30-day standardised risk of mortality) described provision of feedback as important. Other strategies were a high level of respect for emergency medical services as valued professionals/colleagues; employing a hospital-based liaison to deliver training and facilitate communication between pre-hospital and in-hospital teams; and involvement of emergency medical services providers in care improvement initiatives. Provision of quality improvement feedback to paramedics was reported in three further studies $[24,50,51]$ which resulted in better documentation for endotracheal intubation and reduced on-scene times for trauma [51]; increased adherence to pre-hospital protocols for acute stroke [50] and improved door to CT scan times for acute stroke [24].

\section{Discussion}

We did not identify any original research describing the impact on health outcomes from condition-specific structured handover, or from extending the role of ambulance clinicians across the threshold of secondary care for trauma, stroke and MI patients. However, based upon qualitative and observational studies there are promising interventions for further evaluation which could be introduced at different points along the patient care pathway.

The role of ambulance-based paramedics across the threshold of secondary/tertiary care was limited to 'direct transportation' of patients to imaging facilities without further involvement in assessment or treatment, usually in an attempt to improve process measures (door to imaging and door to treatment times). Direct transportation is already a key feature of emergency care planning in the UK National Health Service for trauma, stroke and MI [2]; however there is no such policy initiative for paramedics to apply additional clinical skills during and after handover to hospital personnel.
However, studies with relevance to the further development/ extension of the ambulance-based paramedic role do show that there is potential utility from training paramedics to use standardised communication tools. This was the focus of a previous review [4] which also concluded that evidence favouring structured handover tools (utilising mnemonics) to improve communication during handover is lacking [4]. Consistent with our review there remains insufficient evidence to make any recommendation about condition-specific structured handovers, but if generic formats are helpful then there may be a higher impact from using tailored frameworks which communicate vital information during time-sensitive scenarios. Likewise, condition-specific feedback appears to be of value to paramedics, but is challenging to provide in a timely and structured fashion to a dispersed workforce constantly responding to high service demands.

A range of novel pre-hospital roles for paramedics with enhanced skills (diagnosis, clinical decision making and administration of treatment that was previously the responsibility of hospital physicians) have been developed and implemented successfully [43-47], with improved process outcomes and no additional risk for patients. These enhanced paramedic skills may be directly transferable to working in partnership with hospital ED clinicians across the threshold of secondary care but it was only paramedics already specifically employed to work in a hospital or community healthcare unit who had the opportunity to use the broadest range of skills e.g. as emergency care practitioners (ECP). A literature review of ECPs based at healthcare sites [52] reported benefits in terms of improved patientreported care experience and cost savings, including a reduction in inappropriate referrals to emergency departments following review by community ECPs. Consistent with the current review, strong recommendations were not possible as reports comprised short term retrospective observational reports following recent new investment, and there were no trial evaluations of health outcome.

Given the funding pressures across the entire health care system, it would be prudent to identify how to further capitalise on enhanced paramedic competencies by developing integrated care protocols which focus on health outcomes rather than traditional professional roles and organisational boundaries. The impact of this approach upon outcomes following acute stroke is currently being assessed in the UK by the Paramedic Acute Stroke Treatment Assessment (PASTA) trial [53], which is evaluating the clinical and cost-effectiveness of an enhanced paramedic role before and after admission of patients with suspected stroke.

The main limitation of this review has been reliance upon a narrative description of studies to explore the context surrounding the primary review objective for which no direct evidence was identified. It is also possible that unpublished studies exist which report on the impact of condition-specific structured handover, or an extended 
role of paramedics in hospital on health outcomes for trauma, stroke and MI patients. Eligible studies may have been missed due to excluding non-English language papers and the absence of MeSH terms in the electronic databases for extended paramedic roles.

\section{Conclusions}

Due to the nature of the studies identified, no strong recommendation can be made about changing the handover or post-admission roles of ambulance-based paramedics in hospital for patients with time-critical conditions. However, the literature identified illustrates that paramedic competencies and roles are evolving rapidly and their direct involvement in treatment of patients across the threshold of secondary care in partnership with hospital clinicians has potential to benefit health outcomes. A 'new wave' of paramedic research is needed to inform the design of costeffective handover and feedback processes, and the health impact resulting from enhanced communication and interprofessional sharing of clinical skills.

\section{Appendix 1}

Medline search strategy

1. ambulance*.ti,ab.

2. CCPs.ti,ab.

3. paramedic".ti,ab.

4. (emergency adj3 (service* or technician* or practitioner" or dispatch" or despatch" or triage or communication?)).ti,ab.

5. EMS.ti,ab.

6. emts.ti,ab.

7. ecps.ti,ab.

8. acps.ti,ab.

9. (air adj2 rescue*).ti,ab.

10.HEMS.ti,ab.

11.out-of-hospital.ti,ab.

12.(out adj2 hospital).ti,ab.

13.pre?hospital.ti,ab.

14.tele?medic".ti,ab.

15.(remote adj2 consult*).ti,ab.

16.tele?consult".ti,ab.

17.tele?stroke.ti,ab.

18.or/1-17

19.exp "emergency medical services/

20.exp emergency medical technicians/

21.exp *allied health personnel/

22.exp *Transportation of patients/

23.Emergency medical service communication systems/

24.time-to-treatment/

25.exp emergency service, hospital/

26."telemedicine/

27."remote consultation/

28.or/19-27 29."professional role/

30.Patient Handoff/

31.Patient Care Team/

32.(extend" adj3 (role? or skill? or scope?)).mp.

33.(transfer" adj2 patient?).mp.

34.(role? adj7 (development? or develop or developing or developed)).mp.

35.(role? adj7 impact?).mp.

36.(expand" adj3 (role? or skill? or scope? or responsibilit" or practice?)).mp.

37.((extra or added or additional) adj4 responsibilit").mp.

38.(paramedic* adj3 (skill? or role? or impact? or scope? or practice?)).mp.

39.(emerging adj3 (role? or skill? or scope? or responsibilit* or practice?)).mp.

40.(trends adj7 (paramedic* or role? or skill? or scope?)).mp.

41.(impact? adj9 (paramedic* or role? or skill? or scope?)).mp.

42.(integrat" adj5 (paramedic* or role? or skill? or scope?)).mp.

43.(innovati* adj5 (paramedic* or role? or skill? or scope? or practice?)).mp.

44.(patient" adj4 (handoff or handover or managment)).mp.

45.(advanced adj2 (role? or skill? or scope? or practice?)).mp.

46.(blur* adj4 (boundar* or role?)).mp.

47.(co?operati" adj5 (work or working or role? or team? or practice? or paramedic")).mp.

48.(develop" adj4 responsibilit").mp.

49.((evolution or evolv*) adj3 (practice? or role?)).mp.

50.non-medical.mp.

51.((inter?disciplin* or inter?professional or multi?disciplin* or multi?faceted) adj5 (work or working or role? or team? or staff or practice?)).mp.

52.(substitut" adj2 role?).mp.

53.(medical adj2 substitut*).mp.

54.(new adj3 role?).mp.

55.(scope adj3 practice?).mp.

56.(greater adj2 role?).mp.

57.or/29-56

58.(injury or injuries or injured).mp.

59.infarction?.mp.

60.h?emorrhage.mp.

61.stroke.mp.

62.(collision* or accident").mp.

63.(trauma or traumas or traumatic).mp.

64.poly?trauma.mp.

65.life-threatening.mp.

66.(life adj2 threat").mp.

67.(pre?hospital adj3 (diagnos* or managment or assessment)).mp. 
68.(emergency adj2 patient*).mp.

69.(hospital adj2 door).mp.

70.door-to-needle.mp.

71.door-to-imaging.mp.

72.(emergency adj3 (scan or imaging)).mp.

73.urgent.mp.

74.pre?notif".mp.

75.(priorit" adj2 (pre?hospital or higher or

increased)).mp.

76.(immediacy adj2 (treatment or referral)).mp.

77.rapid transfer.mp.

78.(delay* or timely or urgen* or priorit" or immedia*

or rapid or critical" or sever" or emergenc").mp.

79.exp stroke/

80.critical illness/

81.exp myocardial infarction/

82." "severity of illness index"/

83."emergency medicine/

84.or/58-83

85.(18 or 28$)$ and 57 and 84

\section{Appendix 2}

\section{Study Selection Form}

Table 2 Study selection form

\begin{tabular}{ll}
\hline Study ID: & \\
\hline 1. STUDY DESIGN & $\begin{array}{l}\text { Circle one } \\
\text { response }\end{array}$ \\
RCT, non-randomised trial, quasi-experimental & Yes/No/ \\
(e.g., time series), controlled before-and-after study, & Unsure \\
cohort study, case-control study, cross-sectional &
\end{tabular}

cohort study, case-control study, cross-sectional

study or qualitative study (including mixed methods)

- Exclude if case series or case study

2. PARTICIPANTS

Paramedics/EMTs (or ambulance technicians) in any setting clearly providing a different care process for patients admitted to hospital as an emergency. Include the armed forces but exclude air ambulance or other transportation which includes an accompanying doctor.

\section{INTERVENTION}

Development, evaluation or implementation of novel structured handovers or post-handover clinical activity directly related to patient care in hospital for one

of the following:

- acute stroke

- acute MI

- trauma patients

Exclude non-clinical activity e.g. evaluation of electronic $r$ ecord systems.

\section{OUTCOMES:}

Quantitative assessment of change in a health outcome? Yes/No/

Unsure

Yes for $1,2,3$ AND $4 \rightarrow$ INCLUDE

No for 1, 2, 3 OR $4 \rightarrow$ EXCLUDE (note reason[s] in comments box)

Yes/No/ Unsure

\section{Abbreviations}

CT: Computerised tomography; ECG: Electrocardiogram; ED: Emergency department; EMTs: Emergency medical technicians; IMST

AMBO: Identification, mechanism, injuries, signs, treatment, allergies, medications, background, other; ISBAR: Identification, situation, background, assessment and request; MI: Myocardial infarction; MIST: Mechanism, injury pattern, signs and treatment; NHS: National health service; PASTA: Paramedic acute stroke treatment assessment; PRISMA: Preferred reporting items for systematic reviews and meta-analyses

\section{Acknowledgements}

We would like to express our thanks to Deborah Jones and Liam Dale for their excellent administrative support. GAF is supported by an NIHR Senior Investigator award.

\section{Funding}

This review summarises independent research funded by the National Institute for Health Research (NIHR) under its Programme Grant for Applied Research Programme (RP-PG-1211-20012). The views expressed are those of the author(s) and not necessarily those of the NHS, the NIHR or the Department of Health. The NIHR played no role in the design of the study and collection, analysis, and interpretation of data, or in writing the manuscript.

\section{Availability of data and materials}

All data generated or analysed during this study are included in this published article [and its supplementary information files].

\section{Authors' contributions}

$D F, C P$ and RF conceived the study. DF, CP, RF, HR, GAF were involved in the design of the study. SR designed, conducted and collated the search for studies. DF and RF with assistance from CP assessed studies for inclusion. DF and RF extracted data on study characteristics. All authors were involved in interpretation of data and reviewed the manuscript critically for intellectual content, and approved the final manuscript.

\section{Competing interests}

The authors declare that they have no competing interests.

\section{Consent for publication}

Not applicable.

Ethics approval and consent to participate

Not applicable.

\section{Author details}

${ }^{1}$ Institute of Health and Society, Newcastle University, Baddiley-Clark Building, Richardson Road, Newcastle upon Tyne NE2 4AX, United Kingdom. ${ }^{2}$ Institute of Neuroscience (Stroke Research Group), Newcastle University, Newcastle upon Tyne, United Kingdom. ${ }^{3}$ Research Design Service - North East, Institute of Health and Society, Newcastle University, Newcastle upon Tyne, United Kingdom. ${ }^{4}$ College of Medicine, Swansea University, Wales, United Kingdom. ${ }^{5}$ North East Ambulance Service NHS Foundation Trust, Newcastle upon Tyne, United Kingdom. ${ }^{6}$ Oxford University Hospitals NHS Foundation Trust, Oxford, United Kingdom.

Received: 1 June 2016 Accepted: 17 February 2017

Published online: 23 February 2017

References

1. Keogh B. Review into the quality of care and treatment provided by 14 hospital trusts in England: overview report. 2013. www.nhs.uk/NHSEngland/ bruce-keogh-review/Documents/outcomes/keogh-review-final-report.pdf.

2. NHS England. High quality care for all, now and for future generations: Transforming urgent and emergency care services in England - Urgent and Emergency Care Review End of Phase 1 Report. 2013. http://www.nhs.uk/ NHSEngland/keogh-review/Documents/UECR.Ph1Report.FV.pdf.

3. NHS Confederation. Zero tolerance - making ambulance handover delays a thing of the past. [Online]. 2012. Available at: http://www.nhsconfed.org/ / media/Confederation/Files/Publications/Documents/Zero_tolerance061212. pdf. Accessed 5 Dec 2015. 
4. Wood K, Crouch R, Rowland E, et al. Clinical handovers between prehospital and hospital staff: literature review. Emerg Med J. 2015;32(7):577-81.

5. ledema R, Ball C, Daly B, et al. Design and trial of a new ambulance-toemergency department handover protocol: 'IMIST-AMBO'. BMJ Qual Saf. 2012;21(8):627-33.

6. Flynn D, Francis R, Lally J, et al. A systematic review of paramedics in hospital for acute stroke, acute myocardial infarction and trauma patients. PROSPERO. 2014:CRD42014010785 Available from: http://www.crd.york.ac. uk/PROSPERO/display_record.asp?ID=CRD42014010785.

7. Moher D, Liberati A, Tetzlaff J, et al. Preferred reporting items for systematic reviews and meta-analyses: the PRISMA statement. PLoS Med. 2009;6: e1000097.

8. Higgins JPT, Green S, editors. Cochrane handbook for systematic reviews of interventions version 5.0.2. The Cochrane Collaboration; 2009. Available from http://handbook.cochrane.org/. Accessed 3 Mar 2011.

9. Sterne JAC, Higgins JPT, Reeves BC, on behalf of the development group for ACROBAT-NRSI. A Cochrane Risk Of Bias Assessment Tool: for NonRandomized Studies of Interventions (ACROBAT-NRSI), Version 1.0.0, 24 September 2014. Available from http://www.riskofbias.info. Accessed 11 Nov 2014.

10. El-Masri S, Saddik B. An emergency system to improve ambulance dispatching, ambulance diversion and clinical handover communication-a proposed model. J Med Syst. 2012;36(6):3917-23.

11. Murray SL, Crouch R, Ainsworth-Smith M. Quality of the handover of patient care: a comparison of pre-Hospital and Emergency Department notes. Int Emerg Nurs. 2012;20(1):24-7.

12. Jensen $\mathrm{J}$, Travers $A H$, Bassett $R$, et al. Implementation and operation of a novel paramedic-long term care program. CJEM. 2012;14:S13-4.

13. Young J. I MIST AMBO - a minimum data set to improve clinical handover between paramedics and emergency department physicians. Abstracts of the 2011 Paramedics Australasia Conference - Australasian Journal of Paramedicine. 2013;10(2). Available from https://ajp.paramedics.org/index. php/ajp/article/view/26/31.

14. Jensen $J$, Travers $A H$, Marshall EG, et al. Insights into the implementation and operation of a novel paramedic long-term care program. Prehosp Emerg Care. 2014;18(1):86-91.

15. Jensen $J$, Travers AH, Bardua DJ, et al. Transport outcomes and dispatch determinants in a novel expanded scope paramedic-long-term care program. CJEM. 2013;14:S13.

16. Cummins NM, Dixon M, Garavan C, et al. Can advanced paramedics in the field diagnose patients and predict hospital admission? Emerg Med J. 2013; 30(12):1043-7.

17. Dojmi Di Delupis F, Pisanelli $P$, et al. Communication during handover in the pre-hospital/hospital interface in Italy: from evaluation to implementation of multidisciplinary training through high-fidelity simulation. Intern Emerg Med. 2014;9(5):575-82.

18. Evans SM, Murray A, Patrick I, et al. Clinical handover in the trauma setting: a qualitative study of paramedics and trauma team members. Qual Saf Health Care. 2010;19(6):e57. doi:10.1136/qshc.2009.039073.

19. Meisel ZF, Shea JA, Peacock NJ, et al. Optimizing the patient handoff between emergency medical services and the emergency department. Ann Emerg Med. 2015;65(3):310-7.

20. Scott LA, Brice $J H$, Baker CC, et al. An analysis of paramedic verbal reports to physicians in the emergency department trauma room. Prehosp Emerg Care. 2003;7(2):247-51.

21. Chan EW, Taylor SE, Marriott J, et al. An intervention to encourage ambulance paramedics to bring patients' own medications to the ED: impact on medications brought in and prescribing errors. Emerg Med Australas. 2010;22(2):151-8.

22. Waßmer R, Zimmer M, Ackermann H. Communication management in emergency medicine. Resuscitation. 2010;1:\$89.

23. Ebben RHA, van Grunsven PM, Moors ML, Aldenhoven P, de Vaan J, van Hou R, van Achterberg T, Vloet LCM. A tailored e-learning program to improve handover in the chain of emergency care: a pre-test post-test study. Scand J Trauma Resuscitation Emerg Med. 2015;23:33. doi:10.1186/ s13049-015-0113-3.

24. VonCannon S, Porcelli T. Stroke Systems of Care: EMS Partnerships and Feedback. Stroke. 2016;47(Suppl 1):ATMP81 http://stroke.ahajournals.org/ content/47/Suppl_1/ATMP81.

25. Todt T, Thylen I, Alfredsson J, et al. Strategies to reduce time delays in patients with AcuTe coronary heart disease treated with primary $\mathrm{PCl}$-the
STOP WATCH study: a multistage action research project. BMJ Open. 2013; 3(9):e003493.

26. Pathak R, Cox L, Marley P, et al. Pre-hospital diagnosis of STEMI and impact on door to balloon time (TCH-AMI study). Heart Lung Circ. 2010;19:S147.

27. Binning MJ, Sanfillippo G, Rosen W, et al. The neurological emergency room and prehospital stroke alert: the whole is greater than the sum of its parts. Neurosurgery. 2014;74(3):281-5

28. Colleran R, Mclnerney A, Daly K. Pre-hospital diagnosis of STEMI: Is electronic electrocardiogram transmission superior to paramedic catheterisation lab activation. Ir J Med Sci. 2013;182:S386.

29. Dewhurst MJ, McComb JM. Permanent pacing in patients with complete heart block: The potential safety and cost savings of direct transfer from the ambulance service to a pacing centre. Europace. 2012;13 Suppl 4:iv21.

30. Dieker HJ, Liem SS, El Aidi H, et al. Pre-hospital triage for primary angioplasty: direct referral to the intervention center versus interhospital transport. JACC Cardiovasc Interv. 2010;3(7):705-11.

31. Dorsch MF, Greenwood JP, Priestley C, et al. Direct ambulance admission to the cardiac catheterization laboratory significantly reduces door-to-balloon times in primary percutaneous coronary intervention. Am Heart J. 2008; 155(6):1054-8

32. Farshid A, Tan $\mathrm{R}, \mathrm{O}^{\prime}$ Connor $\mathrm{S}$, et al. Direct ambulance transfer for Primary $\mathrm{PCl}$ is associated with shorter ischaemic times and lower mortality. Catheter Cardiovasc Interv. 2014;83:S20.

33. Huang RL, Thomassee EJ, Park JY, et al. Clinical pathway: helicopter scene STEMI protocol to facilitate long-distance transfer for primary PCl. Crit Pathw Cardiol. 2012;11(4):193-8.

34. Kendall JD, Dutta D, Brown E. Reducing delay to stroke thrombolysislessons learnt from the Stroke 90 Project. Emerg Med J. 2013;32:100-4.

35. Larsson G, Holgers KM. Fast-track care for patients with suspected hip fracture. Injury. 2011;42(11):1257-61.

36. Meretoja A, Weir L, Ugalde M, et al. Helsinki model cut stroke thrombolysis delays to 25 minutes in Melbourne in only 4 months. Neurology. 2013; 81(12):1071-6.

37. Rostykus PS, Gross BW, Dauterman KW, et al. In-hospital mortality for paramedic identified ST elevation myocardial infarction patients with prehospital cath lab activation compares favorably with emergency department activations and with the action registry. J Am Coll Cardiol. 2010; 55(10Suppl1):A185.E1732.

38. Birkemeyer RG, Schneider H, Rillig A, Kische S, Akin I, Paranskaya L, Turan G, Jung $W$, Ince $H$, Nienaber $C$. Bypassing emergency rooms in primary $P C I A$ comparison of two different network concepts. Eur Heart J Acute Cardiovasc Care. 2013;2:129-30.

39. Schustereder G, Hörmanseder B, Einsiedler S, Sperl W, Huemer G, Lugmayr H, Topakian R. Helsinki model in Wels: Stroke thrombolysis door-to-needle time cut by 20 minutes in 5 months. Int J Stroke. 2015;10(Suppl 2):146.

40. Ryan D, Craig A, Turner L, et al. Clinical events and treatment in prehospital patients with ST-segment elevation myocardial infarction. Prehosp Emerg Care. 2013;17(2):181-6.

41. Jensen JL, Walker M, Denike D, et al. Paramedic myocardial infarction care with fibrinolytics: a process map and hazard analysis. Prehosp Emerg Care. 2013;17:429-34.

42. Ranchord AM, Prasad S, Matsis $P$, et al. Paramedic-administered prehospital thrombolysis is safe and reduces time to treatment. N Z Med J. 2009; 122(1302):47-53.

43. Mason S, O'Keeffe $C$, Coleman $P$, et al. Effectiveness of emergency care practitioners working within existing emergency service models of care. EMJ. 2007:24:239-43.

44. Mason S, O'Keeffe C, Knowles E, et al. A pragmatic quasi-experimental multisite community intervention trial evaluating the impact of Emergency Care Practitioners in different UK health settings on patient pathways (NEECaP Trial). EMJ. 2012;29(1):47-53.

45. Campbell SG, Petrie DA, Mackinley RP, et al. Procedural sedation and analgesia facilitator - Expanded scope role for paramedics in the emergency department. Aust J Paramed. 2008;6(3). Available from https://ajp. paramedics.org/index.php/ajp/article/view/462.

46. Foster DB, Dufendach JH, Barkdoll CM, et al. Prehospital recognition of AMI using independent nurse/paramedic 12-lead ECG evaluation: impact on inhospital times to thrombolysis in a rural community hospital. Am J Emerg Med. 1994;12(1):25-31.

47. Zempsky WT, Haskell G. Paramedics as allied health care providers in the pediatric emergency department. Pediatr Emerg Care. 1998;14(5):329-31. 
48. von Vopelius-Feldt J, Benger J. Critical care paramedics in England: a national survey of ambulance services. Eur J Emerg Med. 2014;21(4):301-4.

49. Landman AB, Spatz ES, Cherlin E, et al. Hospital collaboration with emergency medical services in the care of patients with acute myocardial infarction: perspectives from key hospital staff. Ann Emerg Med. 2013;61(2): 185-95.

50. Choi B, Tsai D, McGillivray CG, et al. Hospital-directed feedback to Emergency Medical Services improves prehospital performance. Stroke. 2014;45(7):2137-40.

51. O'Connor RE, Megargel RE. The effect of a quality improvement feedback loop on paramedic skills, charting, and behavior. Prehospital Disaster Med. 1994;9(1):35-8.

52. Hill $H$, McMeekin $P$, Price $C$. A systematic review of the activity and impact of emergency care practitioners in the NHS. Emerg Med J. 2014;10:853-60.

53. Ford G, Price C, White P, et al. Promoting Effective And Rapid Stroke Care (PEARS). NIHR Programme Grant for Applied Research (RP-PG-1211-20012) Newcastle Upon Tyne Hospitals, Northumbria Healthcare, North East Ambulance Service and Newcastle University: £1,938,941. May 2014 to April 2019. http://www.ncl.ac.uk/ion/research/neurodegenerative/ncpdproj6/.

\section{Submit your next manuscript to BioMed Central} and we will help you at every step:

- We accept pre-submission inquiries

- Our selector tool helps you to find the most relevant journal

- We provide round the clock customer support

- Convenient online submission

- Thorough peer review

- Inclusion in PubMed and all major indexing services

- Maximum visibility for your research

Submit your manuscript at www.biomedcentral.com/submit 